\title{
Deslocamento do cérebro na craniotomia estereotáxica para lesão superficial
}

\author{
Paulo Thadeu Brainer-Lima*, Alessandra Mertens Brainer-Lima**, Hildo Cirne Azevedo Filho ${ }^{\star \star \star}$ \\ Serviços de Neurocirurgia do Hospital da Restauração e do Hospital Memorial São José do Recife \\ Centro Diagnóstico Multimagem do Recife
}

\section{RESUMO}

Objetivo: demonstrar por um método alternativo o desvio do alvo estereotáxico calculado pela tomografia computadorizada para cirurgia de tumor cerebral superficial. Método: estudo clínico descritivo de 16 pacientes operados para ressecção da lesão cerebral superficial auxiliada por marcação estereotáxica onde um marcador foi colocado na aracnóide próximo ao alvo, através de buracos de trépano, antes da craniotomia completada. A localização da marca na aracnóide foi aferida antes e depois da craniotomia por fotografia digital. Resultados: a diferença entre os alvos antes e depois da craniotomia foi, em média, de 12,3 mm, com desvio maior para tumores secundários quando comparados com os primários ( $p=0,0048)$. Conclusão: a utilização de estereotaxia em cirurgia com alvo superficial pode necessitar de correção em virtude da modificação do alvo após a craniotomia.

\section{PALAVRAS-CHAVE}

Tumor cerebral. Craniotomia estereotáxica. Alvo estereotáxico.

\section{ABSTRACT}

Brain shift in stereotactic craniotomy to superficial lesion

Objective: in this analysis the authors present an alternative method to quantify brain shifts on superficial tumors with target calculated by the stereotactic frame to determine correlations between these shifts and image characteristics of post craniotomy brain distortion. Method: clinical study of 16 patients operated for resection of superficial cerebral tumors aided by image-guidance system, where marker was placed along cortical arachnoids related with lesion before craniotomy is completed. The location of these markers was measured before and after craniotomy by digital photography. Results: for all cases the mean shift of the cortex after dural opening was $12.3 \mathrm{~mm}$. Each tumor group displayed unique patterns of shift, with greater shift at secondary than primary tumors $(p=0.0048)$. Conclusion: the use of stereotactic technique also needs correction for the shift that the target suffers after craniotomy in surgery of superficial tumors.

\section{KEYWORDS}

Cerebral tumor. Stereotactic craniotomy. Stereotactic target.

\section{Introdução}

O tumor cerebral pode ser de difícil localização e delimitação, tornando sua ressecção completa quase impossível em alguns pacientes ${ }^{8}$. A craniotomia estereotáxica ajuda a localizar o tumor, bem como estabelecer seus limites, de forma que seja minimizado o trauma cirúrgico ao cérebro normal, proporcionando um pós-operatório de menor morbidade .

Entretanto, existe uma diferença entre o alvo calculado durante a aquisição da imagem pela tomografia computadorizada e o mesmo alvo durante a cirurgia ${ }^{3}$, principalmente após a retirada do osso e a abertura da dura-máter (Figura 1).

Neste estudo são analisados 16 pacientes operados pela craniotomia estereotáxica para ressecção de lesão cerebral superficial, ou seja, até $3 \mathrm{~cm}$ de profundidade do plano cortical medida nas imagens de ressonância magnética (RM). O marcador foi colocado na aracnóide segundo o alvo sugerido pela estereotaxia, antes de a craniotomia ser completada. A localização foi aferida antes e depois da craniotomia por fotografia digital. As marcas permitiram determinar o movimento do cérebro e avaliar de forma muito

\footnotetext{
*MD, MSc, PhD, Serviço de Neurocirurgia do Hospital da Restauração e Hospital Memorial São José do Recife. **MD Centro Diagnóstico Multimagem do Recife.

***Professor Titular do Departamento de Neurocirurgia da Universidade de Pernambuco.
} 
Figura 1 - Diagrama ilustrativo do efeito provocado pelo desvio no mesmo alvo estereotáxico superficial antes $e$ depois da craniotomia. A correção pode ser feita simplesmente pela colocação de uma ou mais marcas na aracnóide, por buracos de trepano, antes da craniotomia completada. As setas nos desenhos $A$ e B correspondem ao alvo marcado pela estereotaxia.

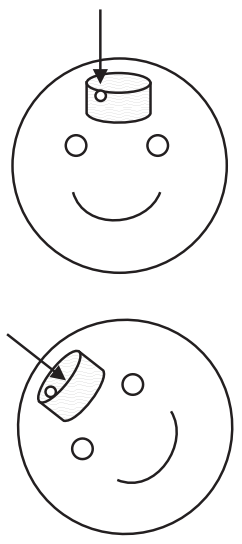

A - Cabeça na posição para realização de neuroimagem

B - Nova posição da cabeça com craniotomia feita e a modificação na posição do encéfalo provocando diferença no mesmo alvo estereotáxico da superfície cortical

simples a distorção entre a imagem e o achado durante a cirurgia.

\section{Pacientes e métodos}

A técnica de colocação de marcas foi utilizada em uma série de 16 pacientes (10 do sexo masculino) com idades entre 33 e 58 anos (média de 42,4 anos), todos operados com índice de Karnofsky ${ }^{5}$ acima de 70 pontos.

O protocolo com todas as informações pertinentes à pesquisa, aos procedimentos, aos riscos e benefícios foi submetido à análise e recebeu aprovação da Comissão Científica e Ética em Pesquisa.

A estereotaxia foi utilizada para definir melhor a localização e o volume da lesão utilizando imagens geradas pela tomografia computadorizada (TC).

Depois da sedação adequada, a moldura de metal compatível (Sistema Estereotáxico TM - Micromar, São Paulo, Brasil) é fixada ao osso, com cuidado para não interferir no acesso à lesão. Segue-se um novo exame de TC, sem e com contraste venoso. Os fiduciais e as coordenadas dos alvos são obtidos de forma padrão ${ }^{2}$. Em todos os pacientes foram definidos o ponto central e os limites do alvo, variando de um a quatro pontos marcados para cada tumor. O local e o tamanho da craniotomia são escolhidos de acordo com a lesão: pequena quando realizada para ressecção simples com microcirurgia; ou grande quando há necessidade de mapeamento cortical.

O mapeamento cerebral de área motora foi realizado em oito pacientes. A estimulação do córtex motor foi feita com paciente sob anestesia geral, sem drogas anestésicas inibidoras da contração muscular, seguindo técnica-padrão ${ }^{1}$.
Antes de completar a craniotomia, alguns orifícios são feitos de acordo com os limites do tumor determinados pela estereotaxia. Esses orifícios são feitos de forma que seja permitida pequena abertura da dura-máter, com pouca ou nenhuma perda de líquido cefalorraquiano. As marcas são colocadas e presas à membrana aracnóide, poupando qualquer outra estrutura adjacente - artéria, veia ou córtex.

As marcas são de titânio, têm 20 mm de comprimento e forma de arco, que se fixam por dobramento, com uma pinça específica (Ligaclip LT 200, ETHICON). Depois da retirada do osso e da abertura completa da dura-máter é feita uma comparação entre o alvo estereotáxico marcado antes e depois da craniotomia completa.

Esse estudo inclui tumores localizados próximos à superfície cortical. Não houve mortalidade. Déficit motor temporário foi encontrado em cinco pacientes com tumor próximo ao córtex eloqüente, operado com auxílio do mapeamento.

\section{Resultados}

A técnica foi empregada com acréscimo de 15 minutos ao tempo cirúrgico. As marcas estavam de acordo com os limites do tumor (15 mm além da margem contrastada da tomografia). O resultado da biópsia nesses pontos realizada em sete pacientes não mostrou tumor em cinco deles.

Foi procurada, com auxílio da fotografia digital, a diferença entre um mesmo alvo de coordenadas determinadas pela estereotaxia, marcadas antes da craniotomia, com um simples orifício de trepanação, e durante a cirurgia, após a craniotomia feita. A diferença entre as marcas antes e após a craniotomia foi sempre maior que $5 \mathrm{~mm}$, em média de $12,3 \mathrm{~mm}$, sendo o desvio máximo de 25 mm identificado em um paciente (Tabela 1).

O desvio do alvo foi maior nos casos de tumores secundários quando comparado ao dos primários $(p=0,0048)$.

\section{Discussão}

A causa para a diferença de posição entre a imagem e o cérebro no transoperatório pode ser fisiológica e mecânica ${ }^{4}$. As fisiológicas são as modificações terapêuticas ou incidentais do fluxo sangüíneo cerebral, principalmente pela administração de diuréticos, ventilação controlada e uso de esteróides. Os fatores mecânicos mais importantes são aqueles relacionados à craniotomia e à posição do paciente pelo efeito da gravidade, perda de líquido cefalorraquiano, distorção ventricular, retração e remoção de tecido cerebral. 
Tabela 1

Características dos pacientes operados e os fatores relacionados à diferença entre os grupos com tumores primário e secundário

\begin{tabular}{|c|c|c|c|}
\hline Variável & Tumor primário & Tumor secundário & Valor de p \\
\hline \multicolumn{4}{|l|}{ Histologia } \\
\hline Astrocitoma & 4 & - & \\
\hline Oligodendroglioma & 3 & - & \\
\hline Cisticercose & - & 2 & \\
\hline Cavernoma & - & 2 & \\
\hline Metástase & - & 4 & \\
\hline Abscesso & - & 1 & \\
\hline \multicolumn{4}{|l|}{ Relação com lobo } \\
\hline Frontal & 3 & 4 & \\
\hline Parietal & 2 & 3 & \\
\hline \multicolumn{4}{|c|}{ Tamanho na RM (cm³) } \\
\hline Médio & 34,2 & 19,4 & \\
\hline Mínimo & 24,0 & 5,0 & \\
\hline Máximo & 45,0 & 28,0 & \\
\hline Desvio-padrão $^{1}$ & 12,2 & 10,44 & $\mathrm{p}=0,0722$ \\
\hline \multicolumn{4}{|l|}{ Edema } \\
\hline Sim & 7 & 7 & \\
\hline Não & - & 2 & \\
\hline \multicolumn{4}{|c|}{ Deslocamento da linha média na RM } \\
\hline Sim & 3 & 5 & \\
\hline Não & 4 & 4 & \\
\hline \multicolumn{4}{|c|}{ Desvio do alvo por diferença entre imagem e cirurgia (mm) } \\
\hline Média & 5,4 & 19,2 & \\
\hline Mínimo & 5,0 & 9,0 & \\
\hline Máximo & 8,0 & 25,0 & \\
\hline Desvio-padrão ${ }^{1}$ & 1,05 & 7,36 & $\mathrm{p}=0,0048^{*}$ \\
\hline
\end{tabular}

*Diferença significativa no nível de 5,0\%.

${ }^{1}$ Por meio do teste $t$-Student para amostras independentes.

A craniotomia estereotáxica exige cautela na identificação de margens em tumores como meningiomas e metástases. Nesses pacientes, manobras terapêuticas para diminuição do edema cerebral no préoperatório, principalmente quando associado a desvio da linha média, podem ampliar o desvio mecânico causado pela craniotomia a ponto de anular os benefícios da marcação estereotáxica.

O implante de esferas radiopacas, cateteres, além da utilização de ultra-sonografia, e ressonância magnética durante a cirurgia ${ }^{7}$ são técnicas para diminuir os efeitos da distorção entre a imagem e o cérebro, principalmente durante a cirurgia para lesões profundas.

A utilização de marcas na superfície cerebral colocada com auxílio da estereotaxia pode auxiliar na ressecção do tumor, sem a necessidade de equipamento adicional $^{2}$. Em alguns pacientes, essas marcas podem anunciar as bordas do tumor, segundo a captação do contraste na TC e, em outros, pode mostrar somente um ponto de referência auxiliar para a ressecção de lesões pequenas e encobertas por córtex de aparência normal.

\section{Referências}

1. BRAINNER-LIMA PT, RAO S, CUKIERT A, GRONISCH G, YACUBIAN EM, MARINO JÚNIOR R: Surgical treatment of refractory epilepsy associated with space occupying lesions, experience and review. Arq Neuropsiquiat 54:384-92, 1996.

2. DEVAUX BC, O'FALLON JR, KELLY PJ: Resection, biopsy and survival in malignant glial neoplasms: $A$ retrospective study of clinical parameters, therapy and outcome. J Neurosurg 78:767-70, 1993.

3. DORWARD NL, ALBERTI O, VELANI B, GERRITSEN FA, HARKNESS WF: Post imaging brain distortion: Magnitude correlates and impact on neuronavigation. J Neurosurg 88:656-62, 1998

4. HASSENBUSCH SJ, ANDERSON JS, PILLAY PK: Brain tumor resection aided with markers placed using stereotaxia guided by magnetic resonance imaging and computed tomography. Neurosurgery 28:801-5, 1991.

5. KARNOFSKY DA, ABELMANN WH, CRAVER LF: The use of nitrogen mustards in palliative treatment of carcinoma. Cancer 1:634-56, 1948.

6. KELLY PJ: Stereotactic resection: general principles. In Kelly PJ (ed): Tumor stereotaxis. Philadelphia, WB Saunders, 1991, pp 268-95.

7. KUROIWA T, OHTA T: Operations using a frameless stereotactic system with a marker: technical note. Min Invasive Neurosurg 44:163-6, 2001.

8. WHITTLE IR: Surgery for gliomas. Curr Opin Neurol 15:663-9, 2002.

Original recebido em abril de 2004

Aceito para publicação em agosto 2004

\section{Endereço para correspondência:}

Paulo Thadeu Brainer-Lima

Rua José Clementino, 47/601

CEP 52050-070 - Recife, PE

E-mail:brainerlima@aol.com 